\title{
Safety and Feasibility of Outpatient Surgery in Benign Prostatic Hyperplasia: a Systematic Review and Meta-Analysis
}

\author{
Stefano Salciccia, MD, PhD, ${ }^{1, *}$ Francesco Del Giudice, MD, ${ }^{1,2, *}$ Martina Maggi, MD, \\ Michael L. Eisenberg, MD, ${ }^{2}$ Benjamin I. Chung, MD, ${ }^{2}$ Simon L. Conti, MD, ${ }^{2}$ Alex M. Kasman, MD, ${ }^{2}$ \\ Fernandino L. Vilson, MD, ${ }^{2}$ Matteo Ferro, MD, ${ }^{3}$ Giuseppe Lucarelli, MD, PhD, ${ }^{4}$ Pietro Viscuso, MD, \\ Giovanni Di Pierro, MD, ${ }^{1}$ Gian Maria Busetto, MD, PhD ${ }^{1}$ Marta Luzi, MD, ${ }^{5}$ Isabella Sperduti, BS, ${ }^{6}$ \\ Gian Piero Ricciuti, MD, ${ }^{1}$ Ettore De Berardinis, MD, ${ }^{1, \dagger}$ and Alessandro Sciarra, MD, $\mathrm{PhD}^{1, \dagger}$
}

\begin{abstract}
Purpose: Most of the endourologic procedures along the urinary tract have been widely practiced as outpatient operations, including surgery for benign prostatic hyperplasia (BPH). This systematic review and meta-analysis was conducted to assess safety and feasibility of outpatient surgery for patients suffering from symptomatic $\mathrm{BPH}$ candidate for endoscopic disobstruction.

Materials and Methods: PubMed, Web of Science, Cochrane, and Embase were searched up until March 30, 2020. Methodological index for nonrandomized studies (MINORS) tool was utilized to assess the quality of included studies, and a pooled measure of failure rate (FR) or event rate (ER) estimate was calculated. Further sensitivity analysis, subgroup analysis, and meta-regression were conducted to investigate contribution of moderators to heterogeneity.

Results: Twenty studies with a total of 1626 patients treated according to outpatient criteria for endoscopic BPH surgery were included. In total, 18 studies reporting data on immediate hospital readmission and/or inability to discharge after endoscopic procedure presented FR estimates ranging from $1.7 \%$ to $51.1 \%$. Pooled FR estimate was 7.8\% (95\% confidence interval [CI]: 5.2-10.3); Heterogeneity: $Q=76.85$; degree of freedom $=17$, $p<0.001 ; I^{2}=75.12 \%$. Subgroup analysis according to surgical technique revealed difference among the three approaches with pooled FR of 3\% (95\% CI: 1-4.9), 7.1\% (95\% CI: 3.9-10.4), and 11.8\% (95\% CI: 7-16.7) for transurethral resection of the prostate, Green-light, and holmium laser vaporesection, respectively $(p<0.001)$. At meta-regression analysis, none of the retrieved covariates was able to significantly influence the cumulative outcomes reported. ER for postoperative complications and early outpatient visit showed a pooled estimate of $18.6 \%$ (95\% CI: $13.2-23.9$ ) and 7.7\% (95\% CI: 4.3-11), respectively.

Conclusions: Our analysis revealed how transurethral procedures for BPH on an outpatient setting are overall reliable and safe. Of note, there were significant outcome differences between groups with regard to type of surgical procedure, perioperative prostate volume, and discharge protocol suggesting the need for further prospective analysis to better elucidate the best strategy in such outpatient conduct.
\end{abstract}

Keywords: outpatient surgery, benign prostatic hyperplasia, laser surgery, HoLEP, TURP, Green-light

\footnotetext{
${ }^{1}$ Department of Maternal Infant and Urologic Sciences, "Sapienza" Rome University, Policlinico Umberto I Hospital, Rome, Italy.

${ }^{2}$ Department of Urology, Stanford University School of Medicine, Stanford, California, USA.

${ }^{3}$ Department of Urology, European Institute of Oncology (IEO), IRCCS, Milan, Italy.

${ }_{5}^{4}$ Department of Emergency and Organ Transplantation-Urology and Kidney Transplantation Unit, University of Bari, Bari, Italy.

${ }^{5}$ Department of Cardiovascular Surgery, Respiratory Medicine, Nephrology, Anesthesiology and Geriatrics, Policlinico Umberto I, Rome, Italy.

${ }^{6}$ Biostatistical Unit, IRCCS Regina Elena National Cancer Institute, Rome, Italy.

*These two authors contributed equally.

${ }^{\dagger}$ These two authors contributed equally.
} 


\section{Introduction}

$\mathbf{L}$ OWER URINARY TRACT symptoms (LUTS) caused by benign prostatic hyperplasia (BPH) can be bothersome and negatively impact on a patient's quality of life. ${ }^{1}$ As the prevalence of symptomatic BPH increases with age, the burden on the health care system and society may increase due to the aging population. ${ }^{2-4}$ In recent years, the surgical treatment of BPH has changed significantly both for the use of medical therapy and for the introduction of new technologies such as laser surgery as highlighted by the recent update to the American Urologic Association guidelines. ${ }^{5}$ Despite several trials which have demonstrated that medications monotherapy or in combination have decreased the progression of BPH and the need for surgery, endoscopic management of BPH remains one of the most performed surgeries in Urology both in an inpatient and outpatient setting. ${ }^{6}$

Due to advancement in technology, many of endourologic procedures developed for BPH have been widely practiced as outpatient operations. ${ }^{7}$ This has been, in part, due to the miniaturization of the instruments and new laser energies available, which has allowed for a reduction in the costs related to the procedure and morbidity. Professional Urologic societies suggest the possibility of managing BPH surgery in an outpatient setting; however, there are no specific recommendations ${ }^{5,8}$ mainly due to the absence of high-quality evidence about the safety of urologic procedures in ambulatory basis and the right population for outpatient procedures. Currently there is a gap in evidence supporting this approach.

In inpatient setting, scientific societies strongly recommend as a reference treatment transurethral resection of the prostate (TURP) and laser surgery, including holmium laser vaporesection (HoLEP) and $532 \mathrm{~nm}$ ("Green-light") laser vaporesection of the prostate with the potassium-titanyl-phosphate and the lithium triborate. ${ }^{5,8}$ All these procedures are effective treatments for moderate-to-severe LUTS secondary to $\mathrm{BPH}$, but they show some differences in terms of safety and required experience. ${ }^{9,10}$ These differences raise the question of whether all these procedures can be valid for an outpatient setting. The aim of this systematic review was to assess the safety and feasibility of outpatient surgery in BPH.

\section{Methods}

This systematic review and meta-analysis was conducted according to Preferred Reporting Items for Systematic Reviews and Meta-Analyses (PRISMA) guidelines. ${ }^{11} \mathrm{~A}$ research question was established based on the Patient-Index Comparator-Outcome-Study design criteria as the following: what is the cumulative failure rate for endoscopic surgical treatment of $\mathrm{BPH}$ in the setting of an outpatient recovery? Furthermore, our goal was to compare current evidence within available retrospective and prospective cohort studies. In particular, we determined the pooled failure rate (FR) for outpatient endoscopic surgical BPH procedure in outpatient setting and the prevalence (event rate [ER]) of postoperative complications and emergency room (Er) readmission/outpatient visit within 30-90 days after the surgery.

\section{Evidence acquisition}

A systematic literature search was performed on PubMed, Embase, Web of Science, and Cochrane up to March 30,
2020. The following search strategies were used: Ambulatory Surgical Procedures OR outpatient OR day case OR ambulatory AND benign prostatic hyperplasia. All titles and abstracts were assessed to select those focusing on outpatient surgery of patients with bladder outlet obstruction secondary to BPH. The references of the included studies were evaluated for other potential trials. In PubMed the function "related articles" were used to research other articles. The full texts of these studies were evaluated by two authors (S.S. and A.S.).

\section{Selection of the studies and criteria of inclusion}

\section{Inclusion criteria}

All control and observational studies independent of their size, language, and status of publication, which included patients who had outpatient surgery for BPH and who reported data on the safety of the procedure.

Only studies which reported outcomes in terms of hospital readmission, Er visit, or ambulatory visit.

Randomized and not-randomized comparative studies if they focused on readmission rate after outpatient surgery. Procedure included: TURP (monopolar, bipolar, plasma resection) or laser surgery, including Thulium laser, Holmium laser, and "Green-light".

The outpatient surgery had to be preplanned as protocol. Only studies where outpatient surgery was defined as $<12$ hours of hospital stay.

\section{Exclusion criteria}

The clinical evaluation after hospital discharge was not clearly reported.

Definition of "outpatient" was not clearly reported.

Outcomes from other minimally invasive therapies (e.g., transurethral incision of the prostate, transurethral thermal ablation of the prostate (needle ablation, microwave therapy, and radiofrequency ablative techniques), prostate stent, and prostatic arterial embolization.

\section{Study quality assessment and statistical analysis}

To assess the risk of bias ( $\mathrm{RoB})$, all included reports were independently reviewed using the "Methodological index for nonrandomized studies" (MINORS), ${ }^{12}$ by assessing the potential risk for selection bias, information bias, measurement bias, or confounding bias. All the studies enrolled were rated using a 12-point scale, according to the method proposed by Slim and colleagues. ${ }^{12}$ Each item was scored from 0 to $2 ; 0$ indicating that it was not reported in the article evaluated, 1 indicating that it was reported but inadequately, and 2 indicating that it was reported adequately. The mean score for each item was then evaluated to compute a cumulative score and to assess the presence of significant differences among the studies enrolled in the review.

Furthermore, publication bias was tested both by visual assessment of the Deeks' funnel plot and calculation of $p$-value using the Deeks' asymmetry test. ${ }^{13}$ The Trim and Fill method was implemented to explore the possible nature of studies "missed" in the review. "We compared each study using ER and $95 \%$ confidence intervals (CIs) for both primary and secondary aim. Sensitivity analyses were performed to assess the contribution of each study to the pooled estimate by 
excluding individual trials one at a time and recalculating the pooled estimates for the remaining studies.

Evaluation for presence of heterogeneity was done using $^{15,16}$ : (1) Cochran's $Q$-test with $p<0.05$ signifying heterogeneity; (2) Higgins $I^{2}$ test with inconsistency index $\left(I^{2}\right)=0 \%-40 \%$, heterogeneity might not be important; 30\%$60 \%$, moderate heterogeneity; 50\%-90\%, substantial heterogeneity; and $75 \%-100 \%$, considerable heterogeneity. The pooled ER estimate was calculated using a random effects model. ${ }^{17}$ Our results are graphically displayed as forest plots, with pooled ERs indicating overall FR for each study implementing an outpatient recovery regimen for endoscopic $\mathrm{BPH}$ treatment and cumulative ER for complications and $\mathrm{Er} /$ outpatient visit.

To explore the predefined outcomes of interest, subgroup analysis was performed regarding differences in surgical technique (TURP, HoLEP, Green-light), preoperative transrectal ultrasound (TRUS) prostate volume (PV) $(<40 \mathrm{cc}$, $>40 \mathrm{cc}$ ), protocol of discharge (with or without urethral catheter), and preoperative America Society of Anesthesiologist (ASA) score (only I-II $v s$ any score). Meta-regression analyses were performed using available continuous demographic and perioperative variables retrieved among the studies to assess potential source of heterogeneity. ERs were plotted against the following available variables: year of publication, mean age of participants, sample size, intraoperative time (minutes), preoperative TRUS volume (cc), catheterization time (hours), volume of resected tissue (cc), and overall MINORS scores.

The point estimates of the $\log$ ERs were obtained and plotted with the area of the circles proportional to the inverse of the squared standard errors of the studies included. Locally weighted scatterplot smoother (LOWESS) function was used to graphically depict the relationship between the different surgical approaches adopted in each single study and the prediction of postdischarge complications and Er/outpatient visit, respectively. Calculations were accomplished using the "metaset" command in Stata version 16.1 (Stata Corporation, College Station, TX) with all tests being two sided and statistical significance set at $<0.05$.

\section{Results}

\section{Search results}

The search strategy identified 807 studies, and 10 additional articles were identified through other sources. After removing the duplicates, 687 studies were screened of which 664 were excluded based on title and abstract. For the remaining 23 studies, the full texts were obtained. Three studies were excluded because they did not meet the inclusion criteria (outpatient definition $>12$ hours). The PRISMA flow diagram is presented in Figure 1. In total 20 records fulfilled the inclusion criteria and were included in the final analysis (Table 1). RoB for the studies included in the qualitative analysis has been reported in accordance with MINORS criteria and is presented in Supplementary Figure S1.

\section{Location, design, and characteristics} of the study population

Twenty studies that met the inclusion criteria were included in this analysis with a total of 1626 patients that were treated on outpatient basis for BPH. The enrollment of pa- tients was performed between 1990 and 2020. The study design was prospective cohort in 14 studies and retrospective in 6 studies. The location of the studies included were North America (9 studies), Europe (8 studies), Australia (1 study), and India (2 study). Data reported included PV (19 studies), ASA score (18 studies), operative time (18 studies), protocol of discharge (with or without catheter, 16 studies), prostatic tissue resected (10 studies), catheter time (13 studies), and type of anesthesia (8 studies).

\section{Technical surgical aspects and perioperative variables assessed}

In terms of type of surgery, four studies were carried out using TURP (one bipolar TURP), nine HoLEP, and six Green-Light (three studies $120 \mathrm{~W}$-HPS, three studies 180 W-KPS). Perioperative outcomes were assessed in terms of outpatient FR (16 studies), Er/outpatient visit (10 studies), and 30-90 days complications-any grade (14 studies).

\section{Cumulative FR of outpatient $B P H$ surgical management}

Only two studies ${ }^{20,36}$ did not report failure events among the patients included in their analysis and therefore were not considered in the quantitative synthesis. In total, 18 studies $^{18,19,21-35,37}$ reporting data on immediate hospital readmission and/or inability to discharge after the endoscopic procedure presented different FR estimates ranging from $1.7 \%$ to $51.1 \%$. Demographic and perioperative characteristics of the 18 studies analyzed are further presented in Table 2. As there was evidence for presence of substantial heterogeneity between the studies: $Q=76.85$ (degree of freedom $[\mathrm{df}]=17), p<0.001 ; I^{2}=75.12 \%$, we reported results according to random-effect model achieving a pooled FR of 7.8\% (95\% CI: 5.2-10.3) (Fig. 2).

According to sensitivity analysis, no single study significantly affected the heterogeneity statistic when removed from the imputation. Inspection of the funnel plot suggested that there was a small-study effect with the smaller studies tending to have higher FR estimates, suggesting the presence of publication bias (Supplementary Fig. S2a). Egger's regression test showed a significant small-study effect $(p<0.001)$, while the "Trim and Fill" method suggested that six "missing" studies would need to be included to remove asymmetry from the funnel plot (Supplementary Fig. S2b). With these hypothetical studies included, the FR having accounted for publication bias was estimated to be $4.8 \%$ (95\% CI 1.9-7.7). However, out of these only two of the six imputed studies would have fallen in the area of statistical significance $(p<1 \%)$ (Supplementary Fig. S2c). None of the variables implemented in the modeling process resulted significantly able in influencing the pooled FR estimate suggesting absence of potential confounders and confident reliability of our findings for the aim of interest. A summary of the meta-regressions performed together with bubble plots is reported in Supplementary Figure S2d.

Subgroup analysis according to type of surgical technique. In total, five studies ${ }^{32-35,37}$ implementing Greenlight, nine articles with HoLEP, ${ }^{23-31}$ and four with TURP $^{18,19,21,22}$ were, respectively, reviewed. Test for differences among subgroups revealed the presence of a 


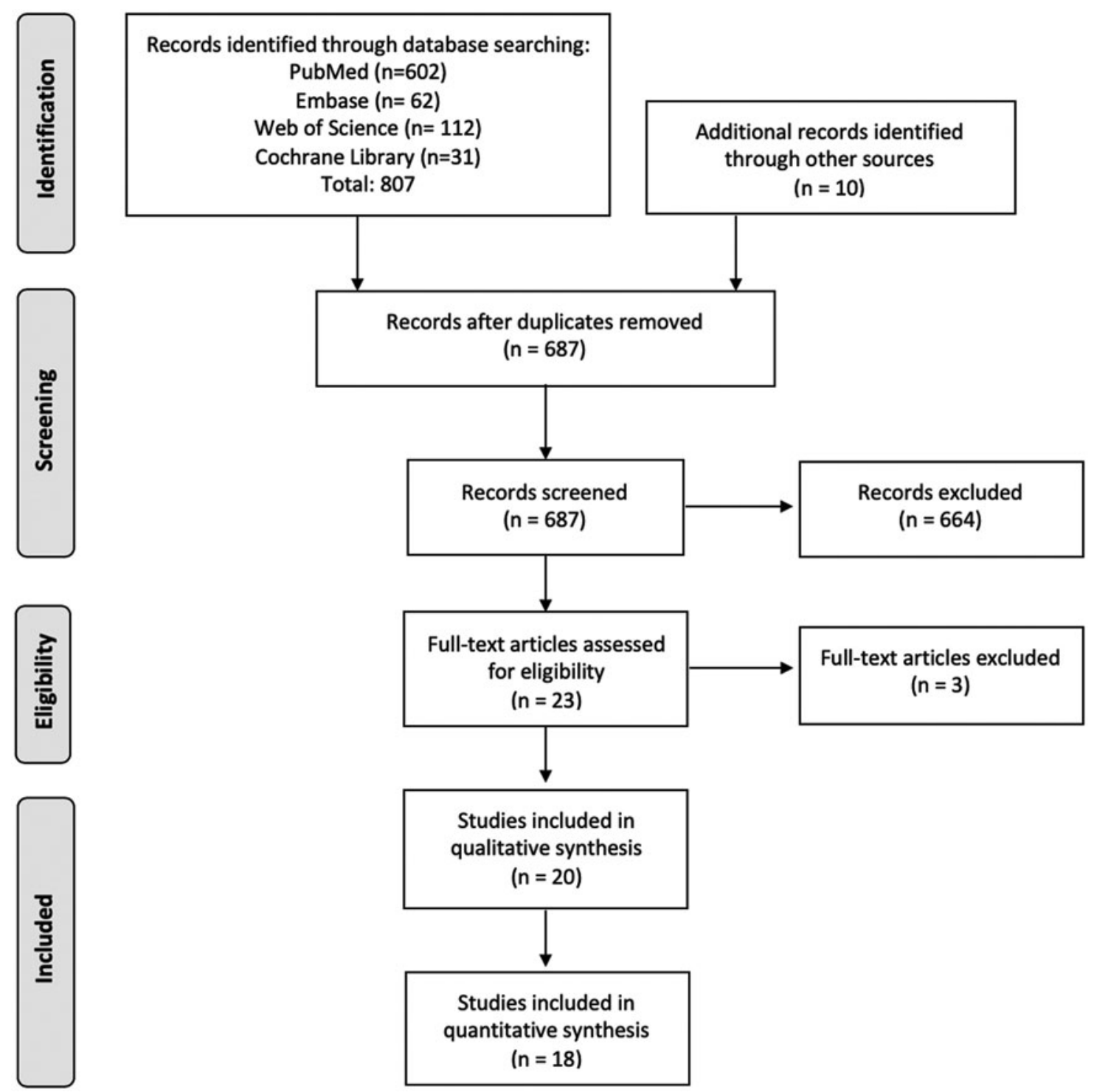

FIG. 1. Preferred Reporting Items for Systematic Reviews and Meta-Analyses flow diagram.

statistical difference among the three different approaches $(Q=13.32$, df $=2, p<0.001)$. Furthermore, the subgrouping reveled evidence for heterogeneity imbalances with the studies implementing HoLEP suffering from higher values $\left(I^{2}: 80.52 \%, Q=56.52, p<0.001\right)$ compared to studies adopting Green-light and TURP, respectively $\left(I^{2}: 34.56 \%\right.$, $Q=7.68, p=0.10$ and $\left.I^{2}: 11.18 \%, Q=4.65, p=0.20\right)$. Pooled FR was 3\% (95\% CI: 1-4.9), 7.1\% (95\% CI: 3.9-10.4), and $11.8 \%$ (95\% CI: 7-16.7) for TURP, Green-light, and HoLEP, respectively (Fig. 3a). As expected, at funnel plot inspection presence of asymmetry distribution was found only in the HoLEP subgroup as depicted in Supplementary Figure S3a.

Subgroup analysis according to preoperative TRUS volume (cc). In total, six studies ${ }^{21-23,25,26,32}$ reporting a preoperative PV $<40 \mathrm{cc}$ and eight ${ }^{24,29-31,33-35,37}$ with $>40 \mathrm{cc}$ were, respectively, enrolled. Test for differences among subgroups revealed presence of a statistical difference among small and larger prostates $(Q=4.88, \mathrm{df}=1, p=0.03)$. Heterogeneity was mainly in the studies with higher $\mathrm{PV}(Q=39.93$, $\left.I^{2}=75.1 \%, p<0.001\right)$. Smaller prostates resulted in lower ER with a pooled FR of $4.1 \%$ (95\% CI: 0.7-7.5) compared to $10.7 \%$ (95\% CI: 6-15.4) within studies with greater PV (Fig. 3b). Funnel plot according to subgroup stratification is presented in Supplementary Figure S3b.

Subgroup analysis according to urethral catheter discharge protocol. Information regarding urethral catheter at discharge was available on 15 studies. Out of these, $11^{18,21,23,25-31,37}$ and 4 studies $^{22,32,33,35}$ reported discharge with and without catheter, respectively. We found that there was evidence for difference among the two subgroups analyzed $(Q=4.5, \mathrm{df}=1, p=0.03)$. Both groups revealed the existence of moderate to substantial $\left(Q=10.99, I^{2}=52.21 \%\right.$, $p=0.01$ and $\left.Q=59.01, I^{2}=79.02 \%, p<0.001\right)$ as also expressed by funnel plot inspection (Supplementary Fig. S3d). 
Table 1. Characteristic of the Studies Included in the Systematic Review and Meta-Analysis

\begin{tabular}{|c|c|c|c|c|}
\hline Author, Location & Study design & $\begin{array}{c}\text { Time of enrollment, } \\
\text { year }\end{array}$ & $\begin{array}{l}\text { Patient with } \\
B P H, \mathrm{n}\end{array}$ & $\begin{array}{c}\text { Managed as } \\
\text { outpatient, } \mathrm{n}(\%)\end{array}$ \\
\hline $\begin{array}{l}\text { McLoughlin and Kinahan }{ }^{18} \\
\text { Canada }\end{array}$ & Prospective cohort & 1987 & 130 & $130(100)$ \\
\hline $\begin{array}{l}\text { Klimberg and associates }{ }^{19} \\
\text { United States }\end{array}$ & Prospective cohort & 1991-1992 & 186 & $125(67)$ \\
\hline $\begin{array}{l}\text { Chander and colleagues }{ }^{20} \\
\text { India }\end{array}$ & Prospective cohort & 2001-2002 & 64 & $64(100)$ \\
\hline $\begin{array}{l}\text { Eaton and Francis }{ }^{21} \\
\text { United Kingdom }\end{array}$ & Prospective cohort & 2001 & 40 & $40(100)$ \\
\hline $\begin{array}{l}\text { Khan } 22 \\
\text { India }\end{array}$ & $\begin{array}{l}\text { Prospective } \\
\text { clinical control trial }\end{array}$ & $2008-2010$ & 120 & $60(50)$ \\
\hline $\begin{array}{l}\text { Larner and associates }{ }^{23} \\
\text { Australia }\end{array}$ & Prospective cohort & 2002 & 38 & $38(100)$ \\
\hline $\begin{array}{l}\text { Gabbay and associates }{ }^{24} \\
\text { France }\end{array}$ & Prospective cohort & 2013-2014 & 30 & $30(100)$ \\
\hline $\begin{array}{l}\text { Jumper and associates } \\
\text { United States }\end{array}$ & Retrospective cohort & 2007-2009 & 65 & $65(100)$ \\
\hline $\begin{array}{l}\text { Ingimarsson and colleagues } \\
\text { United States }\end{array}$ & Retrospective cohort & $2007-2012$ & 66 & $57(86)$ \\
\hline $\begin{array}{l}\text { Lee and associates }^{27} \\
\text { United Kingdom }\end{array}$ & Retrospective cohort & 2013-2016 & 210 & $74(35)$ \\
\hline $\begin{array}{l}\text { Cynk and colleagues }^{28} \\
\text { United Kingdom }\end{array}$ & Prospective cohort & 2011-2012 & 184 & $114(62)$ \\
\hline $\begin{array}{l}\text { Comat and associates } \\
\text { France }\end{array}$ & Prospective cohort & 2012-2015 & 211 & $90(43)$ \\
\hline $\begin{array}{l}\text { Muhsin and colleagues } \\
\text { United States }\end{array}$ & Retrospective cohort & 2017-2018 & 179 & $47(26)$ \\
\hline $\begin{array}{l}\text { Lwin and associates } \\
\text { United States }\end{array}$ & Retrospective control trial & 2013-2018 & 377 & $199(53)$ \\
\hline $\begin{array}{l}\text { Osterberg and colleagues } \\
\text { United States }\end{array}$ & Retrospective cohort & 2011-2013 & 47 & $47(100)$ \\
\hline $\begin{array}{l}\text { Ben-Zvi and associates } \\
\text { Canada }\end{array}$ & Prospective control trial & 2010-2012 & 200 & $120(60)$ \\
\hline $\begin{array}{l}\text { Bowen and colleagues }{ }^{34} \\
\text { Canada }\end{array}$ & Prospective control trial & $2008-2010$ & 164 & $140(85)$ \\
\hline $\begin{array}{l}\text { Berquet and associates } \\
\text { France }\end{array}$ & Prospective cohort & 2012-2013 & 134 & $134(100)$ \\
\hline $\begin{array}{l}\text { Gual and colleagues }{ }^{36} \\
\text { Spain }\end{array}$ & Prospective cohort & 2008-2009 & 50 & $50(100)$ \\
\hline $\begin{array}{l}\text { Corbel and associates }{ }^{37} \\
\text { France }\end{array}$ & Prospective cohort & 2012-2013 & 115 & $115(100)$ \\
\hline
\end{tabular}

Interestingly, pooled FR was higher in patients discharged with urethral catheter $10.2 \%$ (95\% CI: 6.3-14.1) compared with those who were catheter free $(4.4 \%, 95 \%$ CI: $0.8-8)$ (Fig. 3d).

Subgroup analysis according to ASA score distribution. Preoperative recorded ASA scores were identified from 17 studies. ${ }^{18,19,21-33,35,37}$ Out of these, 6 studies $^{18,19,21-}$ 23,29 included only patients with a preoperative score of I or II, while the remaining 11 studies $^{24-33,35,37}$ enrolled patients presenting with any ASA score. Test for homogeneity among the two subgroups demonstrated no significant difference between the adoption of a different ASA score inclusion criterion $(Q=2.03, \mathrm{df}=1, p=0.15)$. Both groups presented substantial heterogeneity as shown in Figure 3c and in Supplementary Figure S3c. FR did not significantly differ among the subgroups with a cumulative estimate of 5.5\% (95\% CI: 2.2-8.8) and 9.2\% (95\% CI: 5.4-13).

\section{ER for postoperative complications and early} outpatient visit

Overall, 6 studies $^{18,19,22,27-29}$ did not report any postoperative complications of any Clavien-Dindo grade, while the remaining 14 experiences accounted for at least one event within a total range of time from 30 to 90 postoperative days. The overall complication estimate ranged from a reported rate of $7.5 \%$ in the study of Eaton and Francis, ${ }^{21}$ as much as $56.7 \%$ in the survey of Gabbay and associates. ${ }^{24}$ Since there was evidence for substantial heterogeneity among the studies $\left(Q=58.26, \mathrm{df}=13, p<0.001 ; I^{2}=74.8 \%\right)$, we relied on a random effect model achieving a pooled ER of $18.6 \%(95 \%$ CI: 13.2-23.9) (Fig. 4a).

According to sensitivity analysis, no single study significantly affected the heterogeneity statistic when removed from the imputation. Inspection of the funnel plot suggested that there was a small-study effect with one smaller study tending to have higher ER estimate. Egger's regression test showed a significant 


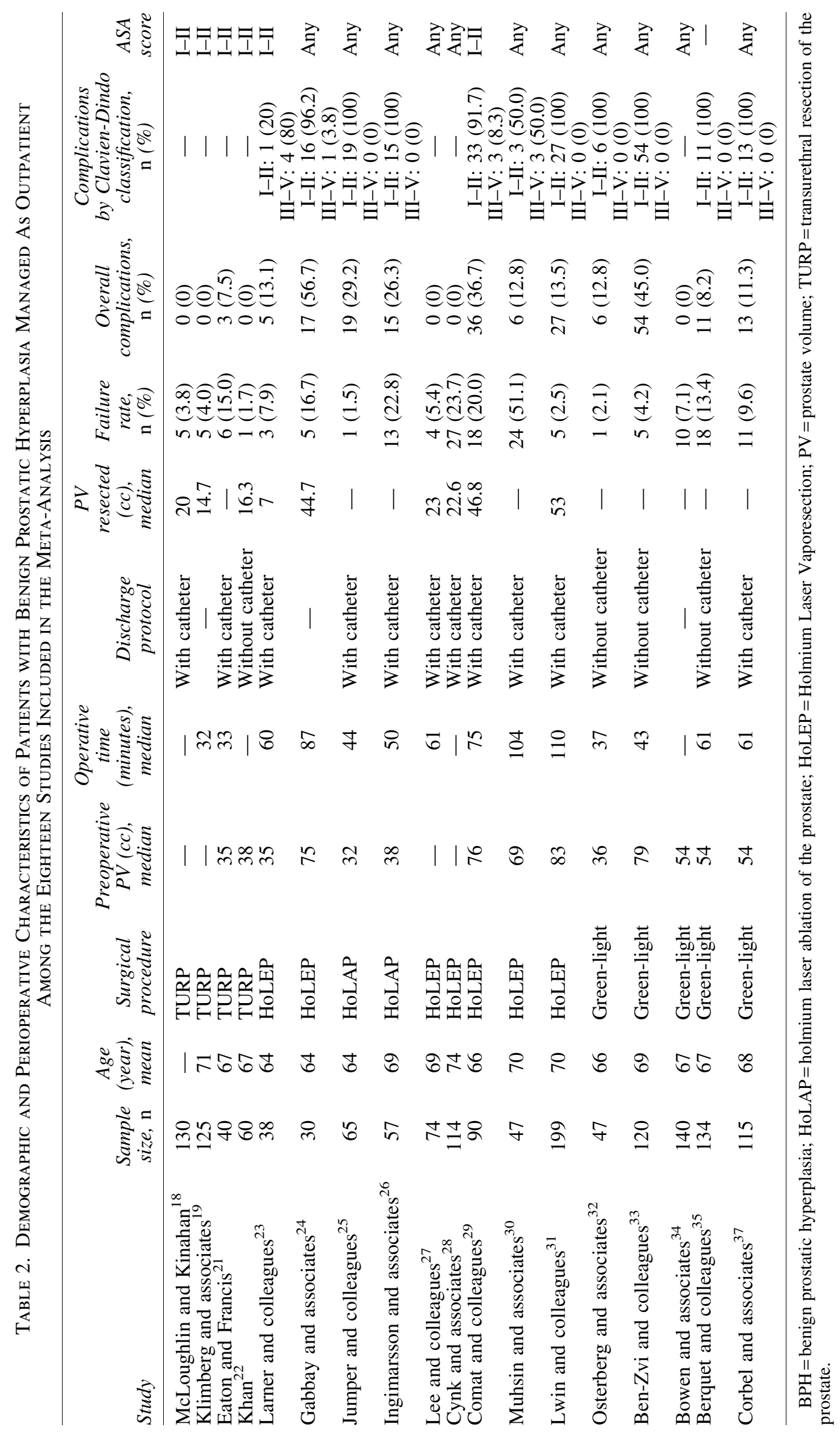




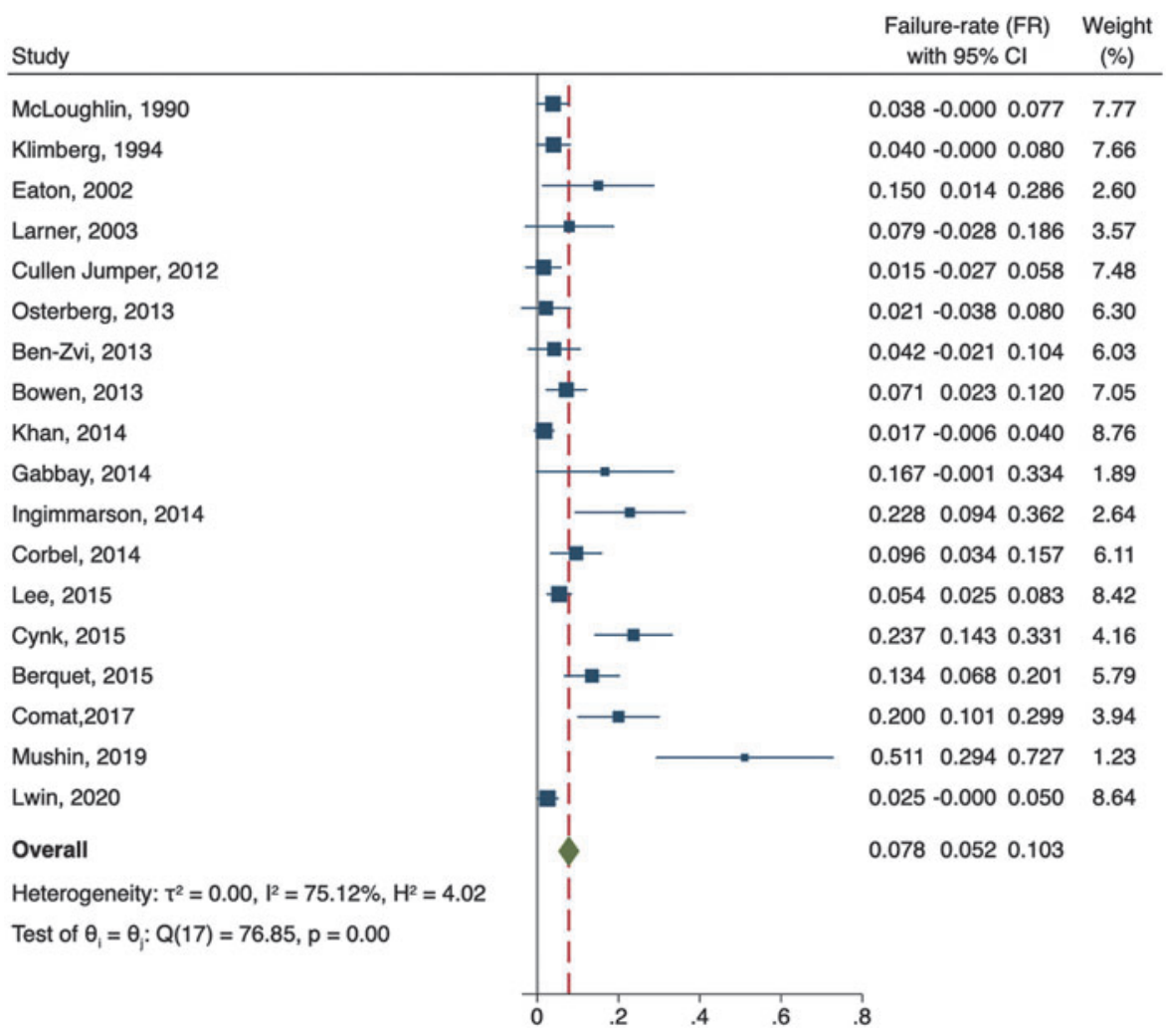

FIG. 2. Forest plot reporting cumulative FR among the 18 studies included in the meta-analysis. $\mathrm{FR}=$ failure rate. small-study effect $(p=0.0001)$, while the "Trim and Fill" method suggested that one single "missing" study would need to be included to remove asymmetry from the funnel plot (Supplementary Fig. S4a). With this hypothetical study included, the FR having accounted for publication bias was estimated to be $17.6 \%$ (95\% CI 12.2-23.1). Subgroup analysis did not reveal the presence of a difference for complication prevalence among the three surgical techniques implemented $(Q=4.57, \mathrm{df}=2, p=0.10)$ nor catheter discharge protocol $(Q=0.25, \mathrm{df}=1, p=0.62)$, as well as for both preoperative mean $\mathrm{PV}$ and ASA score criteria adopted $(Q=0.65, \mathrm{df}=1, p=0.42$ and $Q=0.04, \mathrm{df}=1, p=0.84$ ) (Supplementary Fig. S4b).

At meta-regression none of the continuous variables significantly influenced the aim of interest (Supplementary Fig. S5). Regarding Er/outpatient visit following the procedure, 11 studies did not report any event, while the remaining 9 articles $^{20,21,25,26,29,30,32,34,35}$ showed an ER ranging from $1.5 \%$ in the study of Jumper and colleagues ${ }^{25}$ as much as $11.2 \%$ in the survey of Berquet and colleagues. ${ }^{35}$ The pooled ER using a random-effect model was $7.7 \%$ (95\% CI: 4.3-11) with moderate heterogeneity among the studies $(Q=19$, df $=8, p=0.01 ; I^{2}=50.9 \%$ ) (Fig. $4 b$ ). According to sensitivity analysis, no single study significantly affected the heterogeneity statistic when removed from the imputation. Inspection of funnel plot showed the existence of small-study effect with a significant Egger's regression $(p=0.0009)$. The "Trim and Fill" method suggested that three "missing" studies would need to be included to remove asymmetry from the funnel plot predicting a cumulative ER of 5.9 (95\% CI: 2.5-9.2) (Supplementary Fig. S4c).

At subgroup analysis, none of the aforementioned grouping showed significant differences for the event of interest
(Supplementary Fig. S4d). Of note, intraoperative time (minutes) and increase in PV (cc) showed a significant positive association with ER of Er/outpatient visit (Coeff: 0.002, standard error [SE]: 0.0009, $p=0.024$ and 0.002, SE: 0.0009, $2.17, p=0.03$, respectively) (Supplementary Fig. S6).

Finally, with regard to the surgical technique, despite no significant difference in predicted risk found across all the 20 studies in one approach compared to another for both complication and ER/outpatient visit, we however noticed a rising although slight trend for any complications while a nearly flat predicted distribution of ER/outpatient visit going from the studies using TURP to HoLEP as presented by LOWESS function depicted in Figure 5a and b, respectively.

\section{Discussion}

\section{Overall FR of outpatient surgery for $\mathrm{BPH}$}

Currently, there are two primary management strategies to treating LUTS secondary to BPH: pharmacologic approach or surgical/endoscopic intervention. Despite the proven efficacy of medical therapy in improving symptoms and delaying intervention, endoscopic management of $\mathrm{BPH}$ remains one of the most practiced urologic surgical procedures on both inpatient and outpatient basis. ${ }^{6}$ In recent years due to advancement in technology, most of endourologic procedures along the urinary tract have been widely practiced as outpatient operations, including surgery for BPH. The benefits of outpatient surgery are recognized and mainly include cost savings for health care system and reduced morbidity for the patient. ${ }^{38,39}$ From a methodological point of view, a procedure to be validated for an outpatient setting 
FIG. 3. Forest plot reporting cumulative FR to (a) surgical technique performed (b) preoperative TRUS prostate volume (c) urethral catheter protocol discharge and (d) preoperative ASA score. ASA = America Society of Anesthesiologist; TRUS $=$ transrectal ultrasound . a

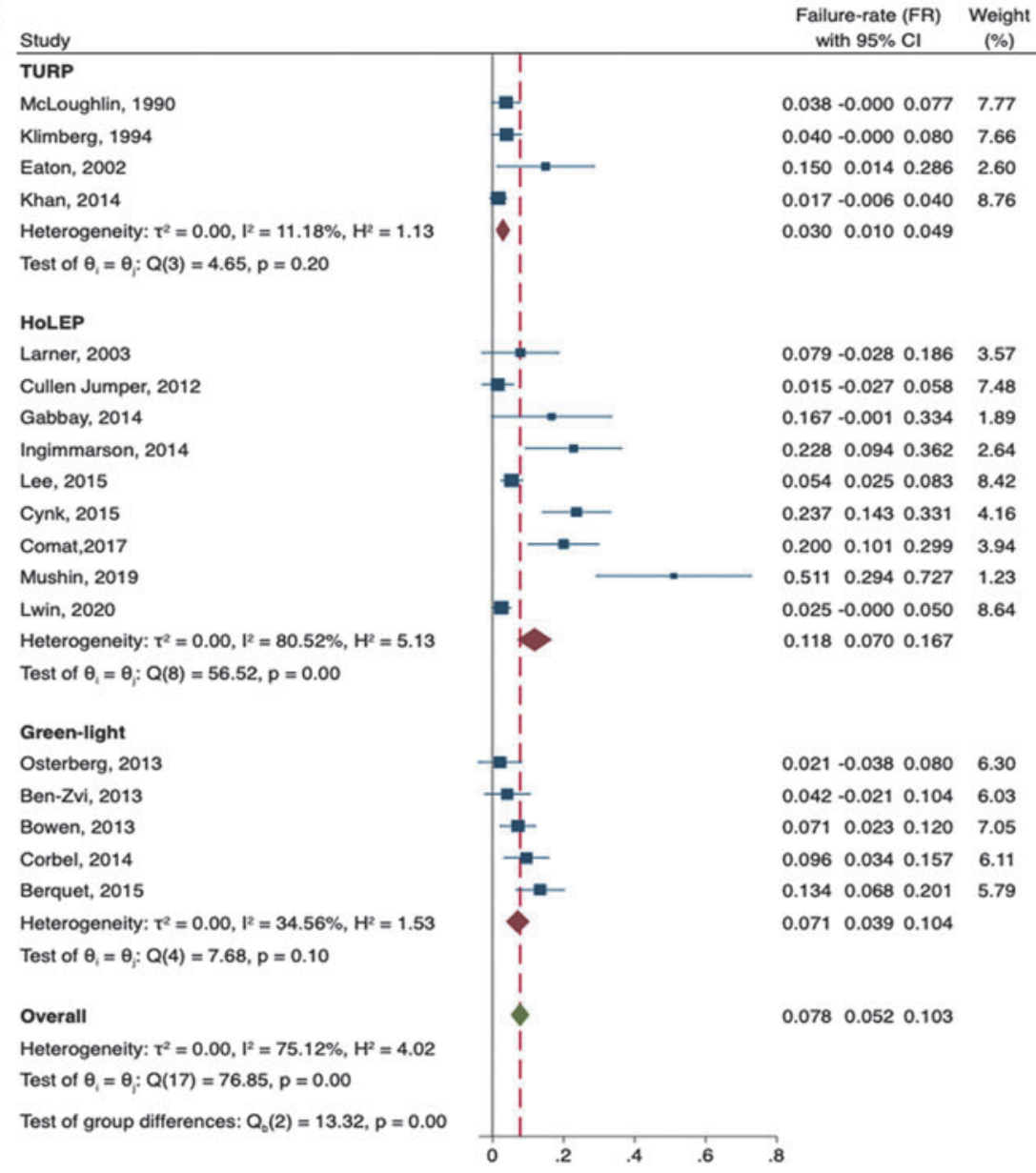

b

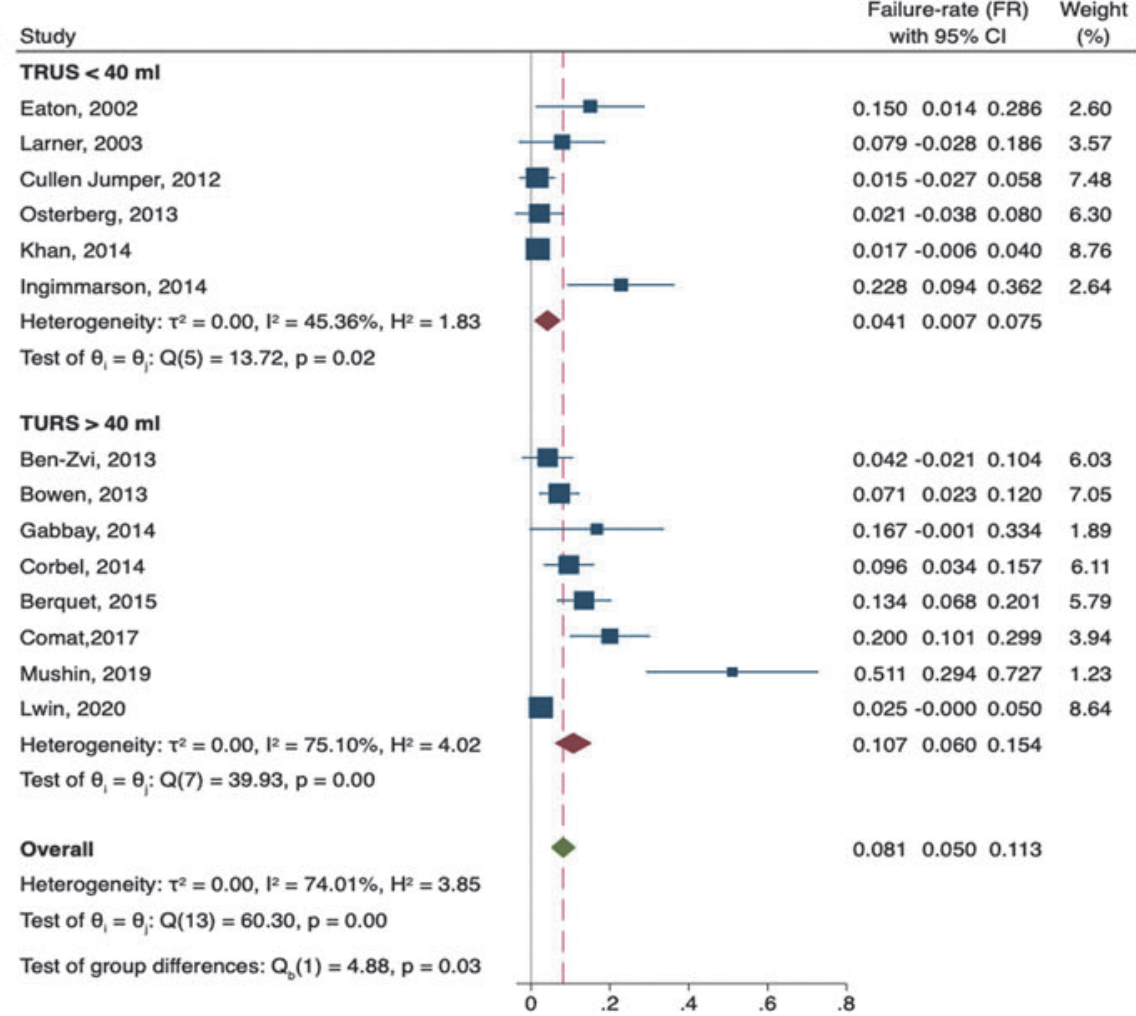

(continued) 
Study

Failure-rate (FR) Weight

Without catheter

Osterberg, 2013

Ben-Zvi, 2013

Khan, 2014

Berquet, 2015

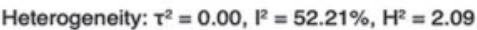

Test of $\theta_{i}=\theta_{i}: Q(3)=10.99, p=0.01$

With catheter

McLoughlin, 1990

Eaton, 2002

Larner, 2003

Cullen Jumper, 2012

Ingimmarson, 2014

Corbel, 2014

Lee, 2015

Cynk, 2015

Comat,2017

Mushin, 2019

Lwin, 2020

Heterogeneity: $\tau^{2}=0.00, \mathrm{I}^{2}=79.02 \%, \mathrm{H}^{2}=4.77$

Test of $\theta_{i}=\theta_{i}: Q(10)=59.09, p=0.00$

Overall

Heterogeneity: $\tau^{2}=0.00,1^{2}=77.92 \%, H^{2}=4.53$

Test of $\theta_{1}=\theta_{i}: Q(14)=73.67, p=0.00$

Test of group differences: $Q_{0}(1)=4.50, p=0.03$

$\begin{array}{lllll}0.021 & -0.038 & 0.080 & 6.30\end{array}$

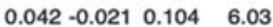

$\begin{array}{llll}0.017 & -0.006 & 0.040 \quad 8.76\end{array}$

$\begin{array}{lllll}0.134 & 0.068 & 0.201 & 5.79\end{array}$

$0.0440 .008 \quad 0.080$

$\begin{array}{lllll}0.038 & -0.000 & 0.077 & 7.77\end{array}$

$\begin{array}{lllll}0.150 & 0.014 & 0.286 & 2.60\end{array}$

$\begin{array}{lllll}0.079 & -0.028 & 0.186 & 3.57\end{array}$

$\begin{array}{lllllllll}0.015 & -0.027 & 0.058 & 7.48\end{array}$

$\begin{array}{lllll}0.228 & 0.094 & 0.362 & 2.64\end{array}$

$\begin{array}{llll}0.096 & 0.034 & 0.157 & 6.11\end{array}$

$\begin{array}{lllll}0.054 & 0.025 & 0.083 & 8.42\end{array}$

$\begin{array}{lllll}0.237 & 0.143 & 0.331 & 4.16\end{array}$

$\begin{array}{llll}0.200 & 0.101 & 0.299 & 3.94\end{array}$

$\begin{array}{lllll}0.511 & 0.294 & 0.727 & 1.23\end{array}$

$\begin{array}{lllll}0.025 & -0.000 & 0.050 & 8.64\end{array}$

0.1020 .0630 .141

$\begin{array}{lll}0.082 & 0.052 & 0.111\end{array}$

d

Study

Failure-rate (FR) Weight with $95 \% \mathrm{Cl}$

(\%)

ASA score 1-II

McLoughlin, 1990

Klimberg, 1994

Eaton, 2002

Larner, 2003

Khan, 2014

Comat,2017

Heterogeneity: $\mathrm{T}^{2}=0.00, \mathrm{l}^{2}=57.00 \%, \mathrm{H}^{2}=2.33$

Test of $\theta_{i}=\theta_{i}: Q(5)=16.62, p=0.01$

Any ASA score

Cullen Jumper, 2012

Osterberg, 2013

Ben-Zvi, 2013

Gabbay, 2014

Ingimmarson, 2014

Corbel, 2014

Lee, 2015

Cynk, 2015

Berquet, 2015

Mushin, 2019

Lwin, 2020

Heterogeneity: $\tau^{2}=0.00, \mathrm{I}^{2}=78.19 \%, \mathrm{H}^{2}=4.58$

Test of $\theta_{i}=\theta_{j}: Q(10)=56.63, p=0.00$

Overall

Heterogeneity: $\mathrm{T}^{2}=0.00, \mathrm{I}^{2}=75.97 \%, \mathrm{H}^{2}=4.16$

Test of $\theta_{i}=\theta_{i}: Q(16)=75.76, p=0.00$

Test of group differences: $Q_{0}(1)=2.03, p=0.15$

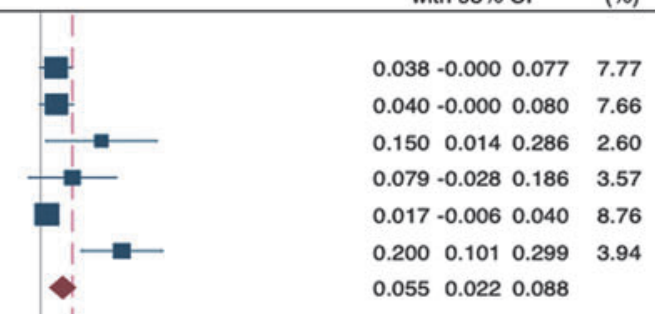

$\begin{array}{llll}0.055 & 0.022 & 0.088\end{array}$

$0.015-0.027 \quad 0.058 \quad 7.48$

$\begin{array}{llll}0.021 & -0.038 & 0.080 & 6.30\end{array}$

$\begin{array}{llll}0.042 & -0.021 & 0.104 & 6.03\end{array}$

$\begin{array}{llll}0.167 & -0.001 & 0.334 & 1.89\end{array}$

$\begin{array}{llll}0.228 & 0.094 & 0.362 & 2.64\end{array}$

$\begin{array}{llll}0.096 & 0.034 & 0.157 & 6.11\end{array}$

$\begin{array}{lllll}0.054 & 0.025 & 0.083 & 8.42\end{array}$

$\begin{array}{llll}0.237 & 0.143 & 0.331 & 4.16\end{array}$

$\begin{array}{llll}0.134 & 0.068 & 0.201 & 5.79\end{array}$

$\begin{array}{llll}0.511 & 0.294 & 0.727 & 1.23\end{array}$

$\begin{array}{lll}0.025 & -0.000 \quad 0.050 \quad 8.64\end{array}$

$0.092 \quad 0.0540 .130$

0.0790 .0520 .106

FIG. 3. Continued. 
FIG. 4. Forest plot reporting pooled ER among the studies assessing (a) any Clavien-Dindo postoperative complication and (b) any emergency room admission/outpatient visit within 30-90 days after the procedure. $\mathrm{ER}=$ event rate.

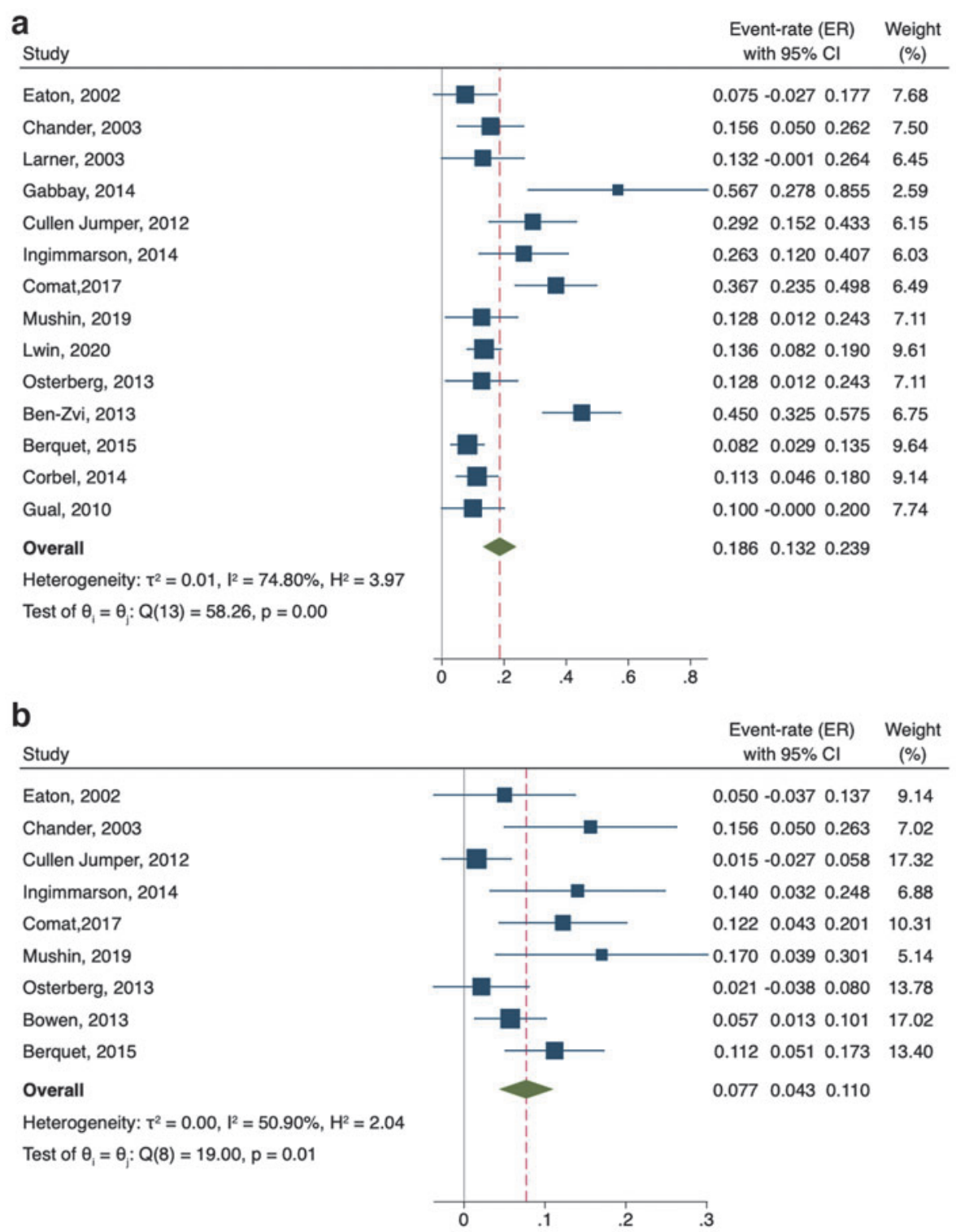

should be safe and able to reproduce the same results as in an inpatient setting.

Among urologic surgical procedures, the endoscopic management of ureteral and kidney stones on outpatient basis is well established and widely practiced worldwide. The widespread use of ambulatory surgery for stone disease could be explained by the lower mean age of the patients and standardization of the technique compared to the surgical complexity of BPH surgery. In the field of BPH surgery, technological advances have resulted in many surgical procedures available with various technologies so that the choice of the device is often linked to surgeon experience and preference. These differences may, in part, explain the low quality of evidences exploring the safety of surgical procedures for $\mathrm{BPH}$ in the outpatient setting and therefore the absence of specific recommendations from urologic societies.

In this systematic review we analyzed the feasibility and safety of outpatient surgery for BPH. We included only those studies with a clear definition of outpatient $(<12$ hours of hospital stay) mainly because this definition reflects the health organization of most countries in the world. For these reasons, studies with a definition of outpatient $<23$ hours, which includes a night of hospitalization, were excluded from the analysis. The meta-analysis showed that outpatient management of BPH is safe with a pooled FR of $7.8 \%(95 \%$ CI: 5.2-10.3). Importantly, none of the variables implemented in the modeling process was able to significantly influence the pooled FR estimate suggesting an absence of potential confounders and confident reliability of our findings for the aim of interest.

Our results are similar to those of inpatient management of BPH using TURP, HoLEP, and Green-light suggesting that from a safety point of view, outpatient surgery achieves the same results as in an inpatient setting. ${ }^{40,41}$ Moreover, our results are similar to those reported by Rambachan and colleagues who found in a large series of urologic outpatient surgical procedures a 30-day hospital readmission rate of $4.27 \%$ for laser prostatectomy and $4.24 \%$ for TURP. ${ }^{42}$ The results of this study, in which a definition of outpatient was 

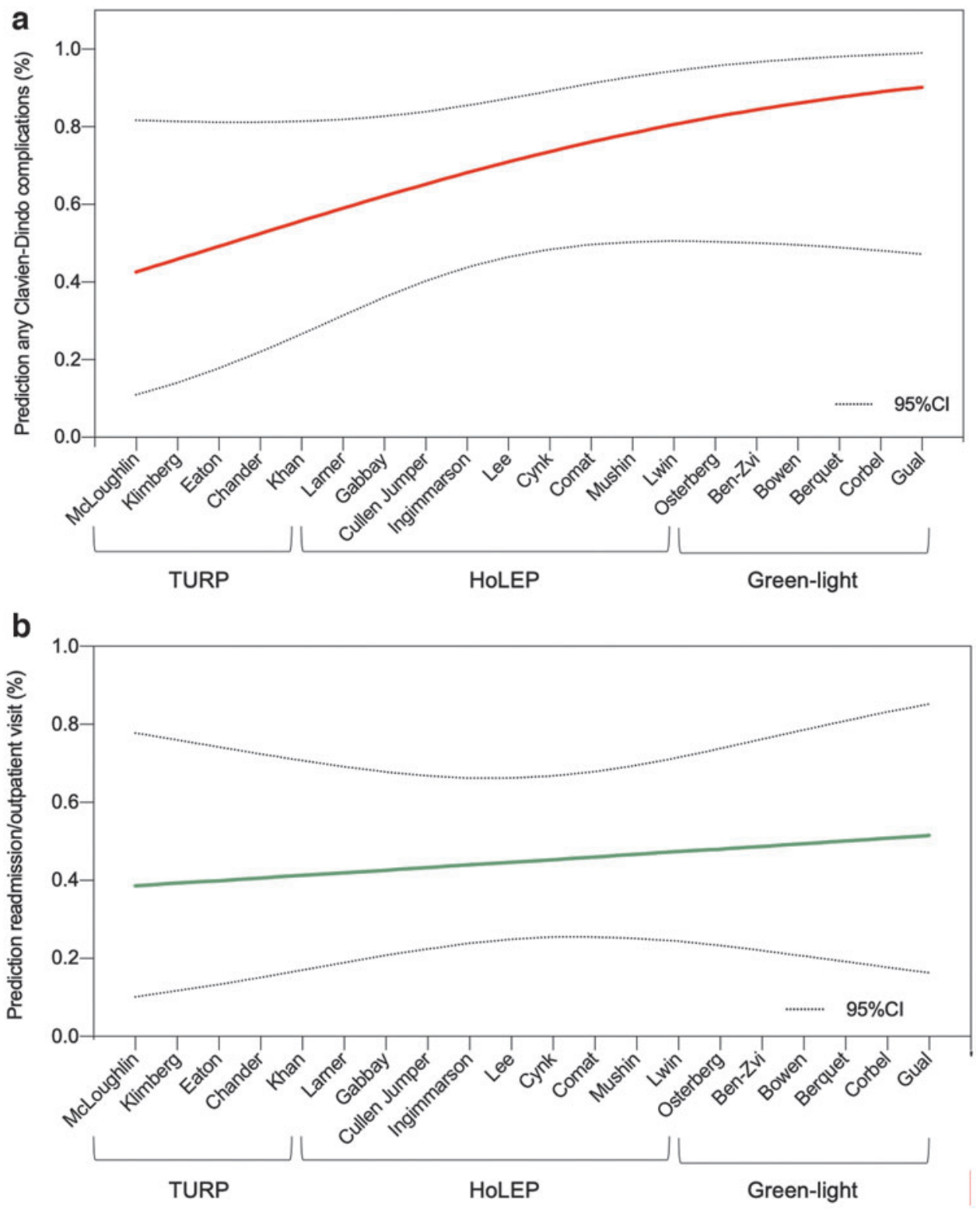

FIG. 5. Locally weighted scatter-plot smoother function depicting the relationship between the different surgical approaches adopted with the prediction of postdischarge complications (a) and emergency room/outpatient visit, respectively, (b) within 30-90 days after the surgery. not reported, indicate that a more stringent definition of outpatient ( $<12$ hours) could result in only a small increase in the rate of readmission $(4.2 \mathrm{vs} 7.8)$.

\section{Factors affecting outpatient FR}

Subgroup analysis showed a statistical difference among the three different approaches $(Q=13.32, \mathrm{df}=2, p<0.001)$ with a pooled FR of 3\% (95\% CI: 1-4.9), 7.1\% (95\% CI: 3.910.4), and $11.8 \%$ (95\% CI: 7-16.7) for TURP, Green-light, and HoLEP, respectively (Fig. 3a). The results of HoLEP are not surprising since this procedure is often performed for larger PVs (selection bias), and in our subgroup analysis PV $>40 \mathrm{cc}$ was associated to worse results in terms of FR $(4.1 \%$ for PV <40 cc vs $10.7 \%$ for PV $>40$ cc). Moreover, compared with Green-light and TURP, HoLEP involves a greater quantity of removed tissue and the enucleation procedure is more complex than TURP and Green-light laser with higher risk of intraoperative complications, including prostatic capsule perforation or complications related to morcellation procedure. ${ }^{43,44}$ Furthermore, among the studies involving the Holmium laser, two studies ${ }^{25,26}$ used an ablation technique (Holmium laser ablation of the prostate [HoLAP]) rather than the enucleation, and we could postulate that such approach may be associated with better safety/tolerability profile than HoLEP. Nevertheless, we did not retrieve any significant difference among this smaller portion of the experiences compared to the others thus not justifying itself the existing heterogeneity with regard to success rate not complication rate within this subgroup.

Another important question is whether the protocol of discharge can affect the rate of hospital readmission. Interestingly in our analysis, pooled FR was higher in patients discharged with urethral catheter $10.2 \%$ (95\% CI: 6.3-14.1), compared with those who were catheter free $(4.4 \%, 95 \% \mathrm{CI}$ : 0.8-8) (Fig. 3c). The protocol of discharge without catheter has been evaluated by several studies mainly using Greenlight laser, and the safety of this protocol has been explained 
by the fact that vaporization reduced scar tissue formation during the healing process and sustained creation of a larger, pliable, and relatively smooth prostatic cavity compared with TURP or other technologies. ${ }^{45}$ In our subgroup analysis according to protocol of discharge, three of four studies involved Green-light so that the result could be explained by this fact. In the field of outpatient surgery the recent advances in anesthetic techniques have progressively led many surgical procedures to be performed on an outpatient basis extending the types of patients eligible for this surgery: for example, the guidelines of the ASAs and other anesthesiologic societies confirm that stable and controlled ASA III or IV is not an absolute contraindication for outpatient surgery for different surgical procedures. ${ }^{46}$ In terms of FR the subgroup analysis according to ASA score showed no significant differences $(Q=2.03, \mathrm{df}=1$, $p=0.15$ ) suggesting that surgical procedures for BPH on outpatient basis are safe both in healthy patients and in patients with comorbidities, including patients taking anticoagulants. ${ }^{26}$

\section{Complications, unplanned visit, and/or Er visit}

As secondary outcomes we analyzed ER for postoperative complications and early outpatient visit or Er visit. The overall complication estimate ranged from a reported rate of $7.5 \%$ in the study of Eaton and Francis ${ }^{21}$ as much as $56.7 \%$ in the survey of Gabbay and associates ${ }^{24}$ with a pooled ER of 18.6\% (95\% CI: 13.2-23.9). Comparing with previous experiences in inpatient setting, the overall rate of complications in our analysis seems to be comparable with other systematic review: Ahyai and coworkers in a meta-analysis of functional outcomes and complications following transurethral procedures reported a rate of perioperative complications from $8.8 \%$ for HoLEP to $24.7 \%$ for Green-light and a similar trend was observed for late complications $(3.5 \%$ for bipolar TURP-25.4\% for Green-light). ${ }^{44}$

In our study the subgroup analysis did not reveal the presence of a difference for complication prevalence among the three surgical techniques implemented $(Q=4.57, \mathrm{df}=2$, $p=0.10$ ). However in terms of outpatient visit/ER visit rate without readmission, our analysis showed that the pooled ER using a random-effect model was 7.7\% (95\% CI: 4.3-11), and with regard to the surgical technique, despite no significant difference in predicted risk of complications or outpatient/ER visits we noticed a slight but interesting rising trend from the studies using TURP to Green-light (Fig. 5a, b). Our analysis showed the existence of consistent variability for HoLEP in terms of reported complications (range $0 \%-$ $56.7 \%$ ) however similar to the complication rate observed within inpatient setting. ${ }^{47,48}$ Although we acknowledge the presence of two out of seven studies adopting the HoLAP technique rather than HoLEP, such discrepancy was not materially or statistically sufficient to justify the existing heterogeneity, which we observed for complication rates involving Holmium laser to treat $\mathrm{BPH}$.

A further possible explanation for these conflicting results might be that the learning curve of HoLEP has historically been indicated as a major barrier to the widespread of the technique and it could be possible that some of the studies enrolled present differences (unfortunately undetectable between the articles) in terms of surgeon's level of expertise and tertiary $v s$ nontertiary centers. This particular aspect is certainly imitating our possible conclusions with regard to
Holmium-based techniques while surely defining the field for future research, which will need to clarify and address this point of concern. To our knowledge, our study represents the first meta-analysis that specifically analyzed safety and feasibility of surgical procedures for BPH in terms of hospital readmission in the specific setting of outpatient surgery.

Our findings suggest that transurethral procedures for $\mathrm{BPH}$ are safe with comparable results with inpatient setting. One of the most important advantages of outpatient surgery is represented by the reduction of the costs related to the procedure as demonstrated by several studies. ${ }^{34,38}$ However, all these studies do not include in their analysis the costs related to unplanned readmission or to Er/outpatient visit. We believe that this aspect should be taken into consideration for future analysis to have an analysis of the costs well balanced between outpatient and inpatient surgery. There are some limitations in our meta-analysis. First, the number of included studies for TURP was small (four studies), and there is some heterogeneity among the included studies. Nevertheless, it included a relatively large number of patients $(n=1626)$ and, therefore, represents the available evidence. Second, we were not able to further analyze surgical experience as per variability of the subgroup analysis so that some of included studies could represent a reference center for specific surgical technique or more generally for the surgical management of patient candidate for BPH interventions.

\section{Conclusion}

In recent years there is increased attention to ambulatory surgery, which has an advantage in terms of costs for health care system and morbidity for the patient. Our analysis showed that transurethral procedures for $\mathrm{BPH}$ in outpatient basis are safe. Subgroup analysis demonstrated significant differences between groups (surgical procedure, PV, protocol of discharge). The degree of heterogeneity between studies limits the strength of our conclusions, but highlights the need for further studies in this field.

\section{Author Disclosure Statement}

No competing financial interests exist.

\section{Funding Information}

No funding was received for this work.

\section{Supplementary Material}

Supplementary Figure S1 Supplementary Figure S2 Supplementary Figure S3 Supplementary Figure S4 Supplementary Figure S5 Supplementary Figure S6

\section{References}

1. Barry MJ. Evaluation of symptoms and quality of life in men with benign prostatic hyperplasia. Urology 2001; 58(6 Suppl 1):25-32.

2. Lee SWH, Chan EMC, Lai YK. The global burden of lower urinary tract symptoms suggestive of benign prostatic hyperplasia: A systematic review and meta-analysis. Sci Rep 2017;7:7984. 
3. Speakman M, Kirby R, Doyle S, et al. Burden of male lower urinary tract symptoms (LUTS) suggestive of benign prostatic hyperplasia (BPH) - focus on the UK. BJU Int 2015;115:508-519.

4. Wei JT, Calhoun E, Jacobsen SJ. Urologic diseases in America project: Benign prostatic hyperplasia. J Urol 2008; 179(5 Suppl):S75-S80.

5. Foster HE, Barry MJ, Dahm P, et al. Surgical management of lower urinary tract symptoms attributed to benign prostatic hyperplasia: AUA guideline. J Urol 2018;200: 612-619.

6. Chughtai BI, Simma-Chiang V, Lee R, Isaacs A, et al. Trends and utilization of laser prostatectomy in ambulatory surgical procedures for the treatment of benign prostatic hyperplasia in New York state (2000-2011). J Endourol 2015;29:700-706.

7. Tabatabaei S, Zangi M. Outpatient surgery for lower urinary tract symptoms/benign prostatic hyperplasia: Truth or myth? Curr Opin Urol 2015;25:27-31.

8. Oelke M, Bachmann A, Descazeaud A, et al. European Association of Urology. EAU guidelines on the treatment and follow-up of non-neurogenic male lower urinary tract symptoms including benign prostatic obstruction. Eur Urol 2013;64:118-140.

9. Mordasini L, Di Bona C, Klein J, et al. 80-W greenlight laser vaporization versus transurethral resection of the prostate for treatment of benign prostatic obstruction: 5Year outcomes of a single-center prospective randomized trial. Urology 2018;116:144-149.

10. Fayad AS, Elsheikh MG, Zakaria T, et al. Holmium laser enucleation of the prostate versus bipolar resection of the prostate: A prospective randomized study. "Pros and Cons". Urology 2015;86: 1037-1041.

11. Liberati A, Altman DG, Tetzlaff J, et al. The PRISMA statement for reporting systematic reviews and metaanalyses of studies that evaluate health care interventions: Explanation and elaboration. J Clin Epidemiol 2009;62: 339.

12. Slim K, Nini E, Forestier D, et al. Methodological index for non-randomized studies (minors): Development and validation of a new instrument. ANZ J Surg 2003;73:712-716.

13. Deeks JJ, Macaskill P, Irwig L. The performance of tests of publication bias and other sample size effects in systematic reviews of diagnostic test accuracy was assessed. J Clin Epidemiol 2005;58:882-893.

14. Shi L, Lin L. The trim-and-fill method for publication bias: Practical guidelines and recommendations based on a large database of meta-analyses. Medicine (Baltimore) 2019;98: e15987.

15. Cohen JF, Chalumeau M, Cohen R, et al. Cochran's Q test was useful to assess heterogeneity in likelihood ratios in studies of diagnostic accuracy. J Clin Epidemiol 2015;68: 299-306.

16. Higgins JP, Thompson SG. Quantifying heterogeneity in a meta-analysis. Stat Med 2002;21:1539-1558.

17. Higgins J, Green S. Cochrane handbook for systematic reviews of interventions version5.1.0. The Cochrane Collaboration available from: www.handbook.cochrane.org .ezproxy.uniroma1.it/chapter_9/9_5_2_identifying_and_ measuring_heterogeneity.htm (last accessed March 2018).

18. McLoughlin MG, Kinahan TJ. Transurethral resection of the prostate in the outpatient setting. J Urol 1990;143:951952 .
19. Klimberg IW, Locke DR, Leonard E, et al. Outpatient transurethral resection of the prostate at a urological ambulatory surgery center. J Urol 1994;151:1547-1549.

20. Chander J, Vanitha V, Lal P, et al. Transurethral resection of the prostate as catheter-free day-care surgery. BJU Int 2003;92:422-425.

21. Eaton AC, Francis RN. The provision of transurethral prostatectomy on a day-case basis using bipolar plasma kinetic technology. BJU Int 2002;89:534-537.

22. Khan A. Day care monopolar transurethral resection of prostate: Is it feasible? Urol Ann 2014;6:334-339.

23. Larner TRG, Agarwal D, Costello AJ. Day-case holmium laser enucleation of the prostate for gland volumes of <60 mL: Early experience. BJU Int 2003;91:61-64.

24. Gabbay G, Bernhard JC, Renard O, et al. Holmium laser enucleation of the prostate as a day case surgery: Prospective evaluation of the first 30 patients. Prog Urol 2015; 25:34-39.

25. Jumper C, Snyder P, Yap RL. Rapid ambulatory pathway laser prostatectomy is safe: Results within the global period. BJU Int 2012;110:1190-1193.

26. Ingimarsson JP, Herrick BW, Yap RL. Ambulatory pathway laser prostate surgery in severely ill patients-feasibility and short-term outcomes. Urology 2014;83:576-580.

27. Lee SM, Gordon K, McMillan R, et al. Day-case holmium laser enucleation of the prostate: Feasibility, safety and predictive factors. Ann R Coll Surg Engl 2018;100:475-479.

28. Cynk M, Georgiadis G, Moore E, et al. Day-case holmium laser enucleation of the prostate. J Clin Urol 2015;8:268273.

29. Comat V, Marquette T, Sutter W, et al. Holmium laser enucleation of the prostate: Prospective evaluation of 90 consecutive cases. J Endourol 2017;31:1056-1061.

30. Muhsin AH, Critchlow W, Navaratnam A, Gnecco J, Tay K, Girardo M, Andrews P, Cheney S, Humphreys M. Feasibility of holmium laser enucleation of the prostate as a 1-day surgery. World J Urol 2020;38:1017-1025.

31. Lwin AA, Zeng J, Evans P, et al. Holmium laser enucleation of the prostate is safe and feasible as a same day surgery. Urology 2020;138:119-124.

32. Osterberg EC, No D, Otto BJ, et al. A retrospective review of office-based 532-nm greenlight laser prostatectomy in men with symptomatic benign prostatic hyperplasia. Urology 2013;82:680-684.

33. Ben-Zvi T, Hueber PA, Liberman D, et al. GreenLight XPS $180 \mathrm{~W}$ vs HPS $120 \mathrm{~W}$ laser therapy for benign prostate hyperplasia: A prospective comparative analysis after 200 cases in a single-center study. Urology 2013;81:853-858.

34. Bowen JM, Whelan JP, Hopkins RB, et al. Photo selective vaporization for the treatment of benign prostatic hyperplasia. Ont Health Technol Assess Ser 2013;13:1-34.

35. Berquet G, Corbel L, Della Negra E, et al. Prospective evaluation of ambulatory laser vaporization of the prostate for benign prostatic hyperplasia. Lasers Surg Med 2015;47: 396-402.

36. Gual BJ, Díez-Caballero AF, García-Miralles GR, et al. [Photovaporization of the prostate with greenlight HPS laser asout patient major surgery] (Spa). Actas Urol Esp 2010;34:170-175.

37. Corbel L, Della Negra E, Berquet G, et al. Ambulatory prostate photo selective vaporization with greenlight laser (180W): Prospective evaluation from 115 patients. Prog Urol 2014;24:733-737. 
38. Nguyen DD, Marchese M, Ozambela M, et al. Ambulatorybased bladder outlet procedures offer significant cost savings and comparable 30-day outcomes relative to inpatient surgery. J Endourol 2020;34:1248-1254.

39. Keoghane SR, Lawrence KC, Gray AM, et al. A doubleblind randomized controlled trial and economic evaluation of transurethral resection vs contact laser vaporization for benign prostatic enlargement: A 3-year follow-up. BJU Int 2000;85:74-78.

40. Zhong J, Feng Z, Peng Y, et al. A systematic review and meta-analysis of efficacy and safety following holmium laser enucleation of prostate and transurethral resection of prostate for benign prostatic hyperplasia. Urology 2019; 131:14-20.

41. Thangasamy IA, Chalasani V, Bachmann A, et al. Photoselective vaporization of the prostate using $80-\mathrm{W}$ and $120-\mathrm{W}$ laser versus transurethral resection of the prostate for benign prostatic hyperplasia: A systematic review with metaanalysis from 2002 to 2012. Eur Urol 2012;62:315-323.

42. Rambachan A, Matulewicz RS, Pilecki M, et al. Predictors of readmission following outpatient urological surgery. J Urol 2014;192:183-188.

43. Cornu JN, Ahyai S, Bachmann A, et al. A systematic review and meta-analysis of functional outcomes and complications following transurethral procedures for lower urinary tract symptoms resulting from benign prostatic obstruction: An update. Eur Urol 2015;67:1066-1096.

44. Ahyai SA, Gilling P, Kaplan SA, et al. Meta-analysis of functional outcomes and complications following transurethral procedures for lower urinary tract symptoms resulting from benign prostatic enlargement. Eur Urol 2010;58: 384-397.

45. Spaliviero M, Araki M, Page JB, et al. Catheter-free $120 \mathrm{~W}$ lithium triborate (LBO) laser photoselective vaporization prostatectomy (PVP) for benign prostatic hyperplasia (BPH). Lasers Surg Med 2008;40:529-534.

46. Association of Anesthetists of Great Britain and Ireland; British Association of Day Surgery. Day case and short stay surgery: 2. Anesthesia 2011;66:417-434.

47. Shah HN, Mahajan AP, Hegde SS, Bansal MB. Perioperative complications of holmium laser enucleation of the prostate: Experience in the first 280 patients, and a review of literature. BJU Int 2007;100:94-101.

48. Kampantais S, Dimopoulos P, Tasleem A. Assessing the learning curve of holmium laser enucleation of prostate (HoLEP). A systematic review. Urology 2018;120:9-22.

Address correspondence to: Francesco Del Giudice, MD

Department of Maternal Infant and Urologic Sciences "Sapienza" Rome University Policlinico Umberto I Hospital Rome 00161 Italy

E-mail: francesco.delgiudice@uniroma.it; fdelgiu@stanford.edu

$\begin{aligned} & \text { Abbreviations Used } \\ \mathrm{ASA} & =\text { America Society of Anesthesiologist } \\ \mathrm{BPH} & =\text { benign prostatic hyperplasia } \\ \mathrm{CI} & =\text { confidence interval } \\ \mathrm{df} & =\text { degree of freedom } \\ \mathrm{Er} & =\text { emergency room } \\ \mathrm{ER} & =\text { event rate } \\ \mathrm{FR} & =\text { failure rate } \\ \mathrm{HoLAP} & =\text { holmium laser ablation of the prostate } \\ \text { HoLEP } & =\text { holmium laser vaporesection } \\ \mathrm{LOWESS} & =\text { locally weighted scatterplot smoother } \\ \mathrm{LUTS} & =\text { lower urinary tract symptoms } \\ \mathrm{MINORS} & =\text { Methodological Index for Nonrandomized } \\ & \quad \text { Studies } \\ \mathrm{PRISMA} & =\text { Preferred Reporting Items for Systematic } \\ \mathrm{PV} & =\text { Reviews and Meta-Analyses } \\ \mathrm{RoB} & =\text { risk of bias } \\ \mathrm{SE} & =\text { standard error } \\ \mathrm{TRUS} & =\text { transrectal ultrasound } \\ \mathrm{TURP} & =\text { transurethral resection of the prostate }\end{aligned}$

